\title{
A cost-effective liquid phase exfoliation process for large 2D-MoS 2 nanosheets and its application in FET
}

Cite as: AIP Conference Proceedings 2265, 030696 (2020); https://doi.org/10.1063/5.0016732

Published Online: 05 November 2020

Rohit Sharma, Mahima Chaudhary, Ashish Kumar, Reena Kumari, Preeti Garg, G. Umapathy, L. Radhapiyari Devi, Sunil Ojha, Ritu Srivastava, and O. P. Sinha

View Online

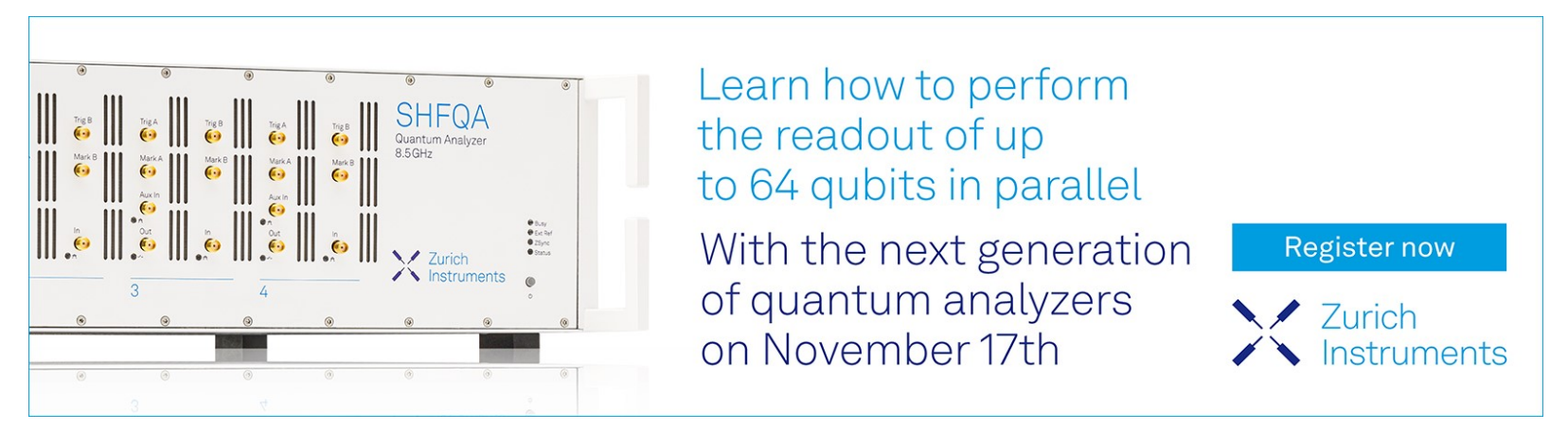




\title{
A cost-effective liquid phase exfoliation process for large 2D- $\mathrm{MoS}_{2}$ nanosheets and its application in FET
}

\author{
Rohit Sharma ${ }^{1}$, Mahima Chaudhary ${ }^{1}$, Ashish Kumar ${ }^{2}{ }^{2 a}$, Reena Kumari ${ }^{2}$, Preeti \\ Garg $^{3}$, G. Umapathy ${ }^{4}$, L. Radhapiyari Devi ${ }^{3}$, Sunil Ojha ${ }^{4}$, Ritu Srivastava ${ }^{2}$ and O. \\ P. Sinha ${ }^{1, a)}$ \\ ${ }^{1}$ Amity Institute of Nanotechnology, Amity University UP, Noida-201301 (UP) India \\ ${ }^{2}$ CSIR- National Physical Laboratory, Dr. K.S. Krishnan Marg, New Delhi-110012,India \\ ${ }^{2 a}$ Academy ofScientific and Innovative Research (AcSIR), Ghaziabad-201002, India \\ ${ }^{3}$ Solid State Physics Laboratory, Timarpur, New Delhi- 110054, India \\ ${ }^{4}$ Inter University Accelerator Centre, Aruna Asaf Ali Marg, New Delhi- 110065, India \\ ${ }^{\text {a)} C o r r e s p o n d i n g ~ a u t h o r: ~ o p s i n h a @ a m i t y . e d u ~}$
}

\begin{abstract}
D-TMDC materials are supposed to be suitable materials for the electronic industry requirements due to tunable bandgap. 2D- $\mathrm{MoS}_{2}$ has an advantage over the graphene as it has direct bandgap and high on/off ratio. In this work, 2D-MoS 2 nanostructures have been synthesized using a simple and cost-effective liquid phase exfoliation (LPE) method in the organic solvent without any additives. The synthesized $\mathrm{MoS}_{2}$ has up to 4-layer thick nanosheets structure which is confirm by the FESEM and Raman studies. From the UV-Visible absorption spectroscopy, the bandgap of the material is found to be $1.79 \mathrm{eV}$. This synthesized material is used as the channel material in the field effect transistor. The field effect transistor (FET) device have been fabricated in the top-gate configuration. It has been found that the current on/off ratio is of the order of $10^{4}$.
\end{abstract}

\section{INTRODUCTION}

Graphene, a wonder material, was considered as one of the best materials to revolutionize the electronic industry due to its superior electronic, optical and mechanical properties. Graphene is the first 2D material which have been explored for many applications. Graphene based devices may have carrier mobility of $\sim 10^{5} \mathrm{~cm}^{2} / \mathrm{Vs}$ and cut off frequency higher than $\sim 100 \mathrm{GHz}$ [1]. But due to its zero bandgap, it is not suited for opto-electronic applications. The bandgap of the graphene can be modified by using different morphology and techniques like nanoribbon or it can be done by adding the dopant to the graphene[2-3]. But all this process affects it carrier mobility and other properties and cannot be suitable for electronic applications. Therefore, a thrust for alternative single/layered structured materials was on air. Layered transition metal dichalcogenides (TMDC) materials have similar structure and certain advantages over the graphene e.g. $2 \mathrm{D}-\mathrm{MoS}_{2}$ has the direct bandgap of 1.8-1.9 eV [4-5]. There are no dangling bonds in the $\mathrm{MoS}_{2}$, and low dimensionality make them more suitable for the electronic and opto-electronic devices. Two-dimensional (2D) materials have attracted a lot of interest in the device's physics. Recently, $\mathrm{MoS}_{2}$ based field effect transistor have been fabricated having the high on/off ratio $10^{8}$ and carrier mobility of $200 \mathrm{~cm}^{2} / \mathrm{Vs}$ [6-7].

In this work, we have synthesized the $2 \mathrm{D}-\mathrm{MoS}_{2}$ nanosheets by a cost-effective liquid phase exfoliation (LPE) method. The synthesized material has been characterizing by the field emission scanning electron microscope (FESEM), UV-Visible absorption spectroscopy and Raman spectroscopy. The synthesized material has been used as the channel material in the Field effect transistor (FET). The FET has been fabricated in the top-gate mode. The fabricated device has been studied by the I-V measurement. 


\section{EXPERIMENTAL METHOD}

Bulk $\mathrm{MoS}_{2}$ powder was purchased from Alfa Aesar ( $<2 \mu \mathrm{m}$, cash no-), n-methyl-2-pyrrolidone (NMP) from Merck. The $\mathrm{MoS}_{2}$ nanosheets are synthesized by the liquid phase exfoliation [8]. The $\mathrm{MoS}_{2}$ and NMP was taken in $10 \mathrm{mg} / \mathrm{ml}$ concentration in glass vial. The prepared solution was ultrasonicated in the bath sonicator at $40 \mathrm{KHz}$ frequency for $8 \mathrm{hr}$. After that the solution is left for overnight and centrifuge at $3000 \mathrm{rpm}$. Later, suspension is collected. The remaining percipate is again ultrasonicated in the fresh NMP for half an hour and centrifuge at high $15000 \mathrm{rpm}$. After that supernant is collected and characterized. The top gate field effect transistor is fabricated with the prepared sample as shown in figure 1 . The channel is pattern on the ITO having width of $0.5 \mathrm{~mm}$ and then the pattern ITO is soap clean for 5 mins. Again ultrasonicated for the $15 \mathrm{~min}$ in the DI-water three times and the sonicated in the acetone for 15 mins after that IPA vapor cleaning is done. Pattern ITO is dry in the vacuum oven at $60 \mathrm{C}$ for 45 mins. After the cleaning process, $\mathrm{MoS}_{2}$ sample is spin coated on the ITO and $120 \mathrm{~nm}$ of LiF is deposited with the thermal evaporation and after the $\mathrm{Al}$ as gate has been deposited.

The characterization tools that have been used to study the structural, optical and electrical properties of $\mathrm{MoS}_{2}$ based FET are Field emission electron microscopy (FESEM), Raman spectrometer, UV-Visible spectrometer, and Semiconductor analyzer.

\section{RESULTS AND DISCUSSION}

The morphological and structural properties of the prepared sample were studied by the FESEM (fig 1a) and Raman Spectroscopy (fig 1b), as shown in the figure 1 (a) and (b) respectively. FESEM reveals the formation of nanosheets having the lateral dimension of as large as few $\mu \mathrm{m}$ and thickness of few layers. From the Raman studies, there are two modes of vibrations appeared in the fig $1(\mathrm{~b})$. The peak at $383.64 \mathrm{~cm}^{-1}$ is related to the in-plane vibration i.e. $\mathrm{E}_{2 \mathrm{~g}}^{1}$ of $\mathrm{MoS}_{2}$ and the at $408 \mathrm{~cm}^{-1}$ is out-plane vibration i.e. $\mathrm{A}_{1 \mathrm{~g}}$. The in-plane mode corresponds to the sulphur atoms vibrating in one direction and the molybdenum atom in the other, while the out-of-plane mode is a mode of just the sulphur atoms vibrating out-of-plane. As $\mathrm{MoS}_{2}$ becomes single layer these two modes evolve with thickness. The in-plane mode upshifts and the out-of-plane downshifts. The difference of these two modes $\left(\sim 18 \mathrm{~cm}^{-1}\right)$ can be used as a reliable identification for monolayer $\mathrm{MoS}_{2}$. In our case, the peak difference is $\sim 24 \mathrm{~cm}^{-1}$, which indicates that the synthesized materials have up to 4 layers [9].
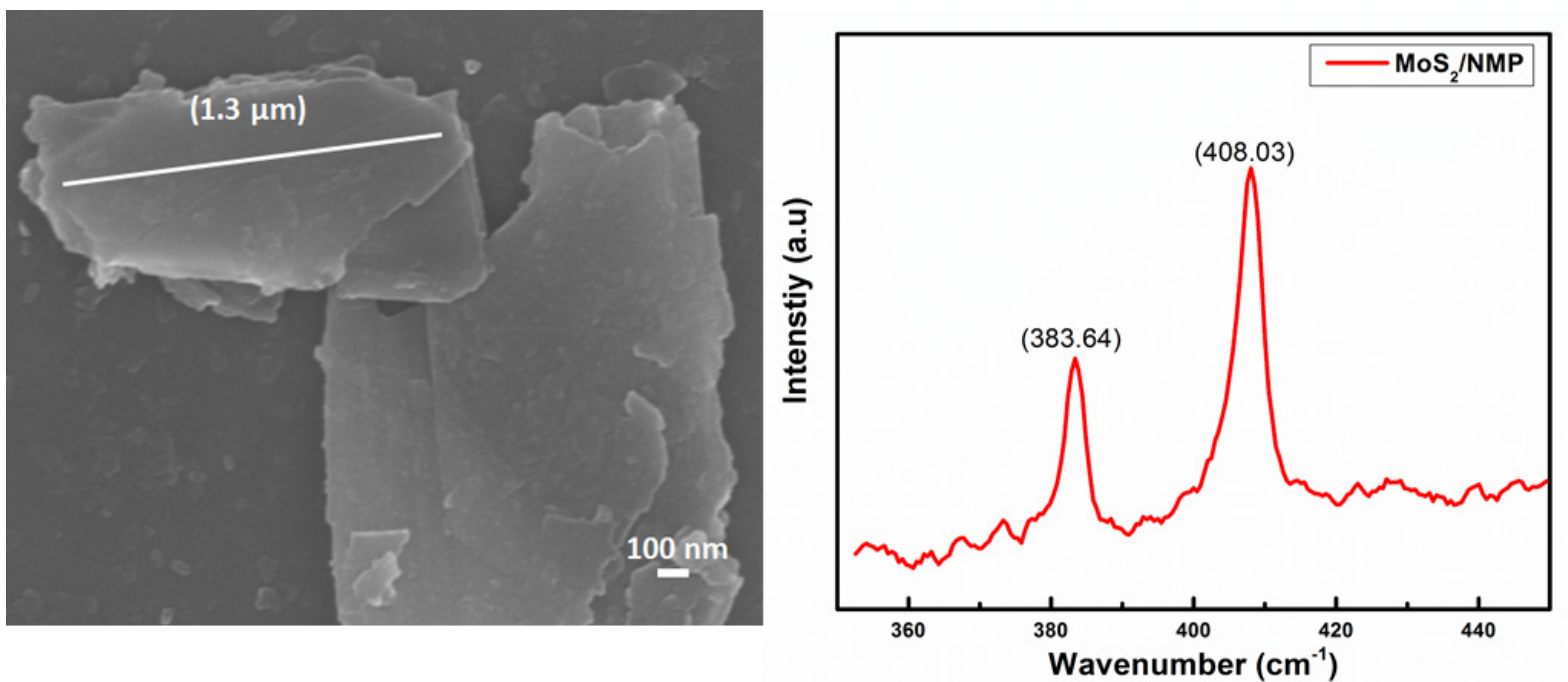

FIGURE 1. ( a) the FESEM image of $\mathrm{MoS}_{2}$ nanosheets and (b) shows the Raman spectrum few layer thick $\mathrm{MoS}_{2}$. 

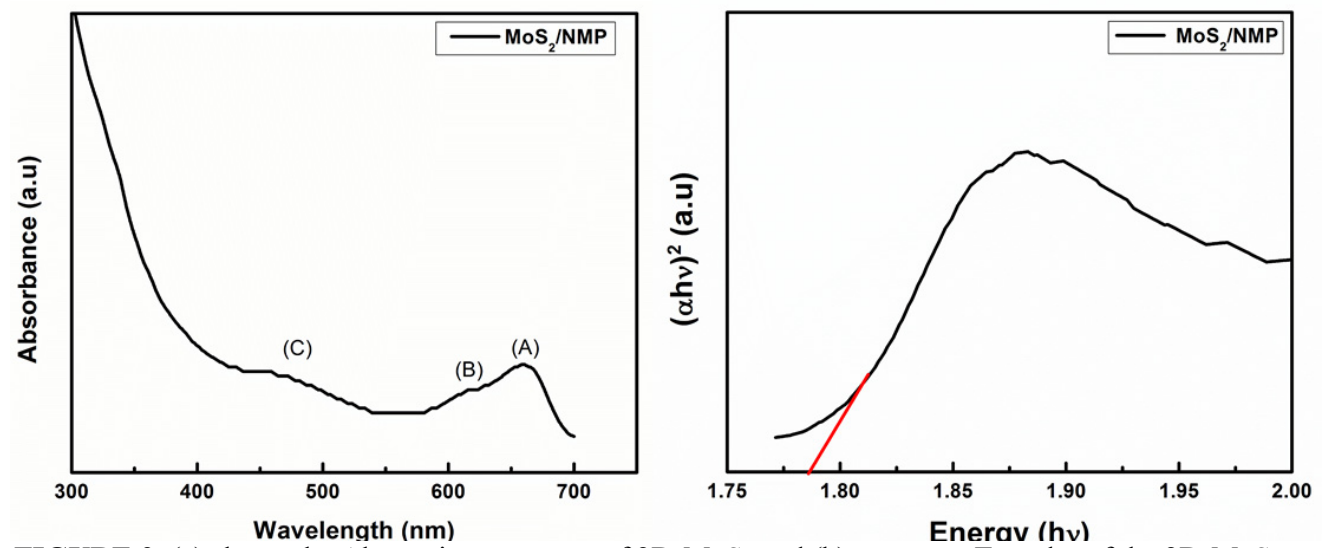

FIGURE 2. (a) shows the Absorption spectrum of $2 \mathrm{D}-\mathrm{MoS}_{2}$ and (b) represent Tau-plot of the 2D-MoS 2
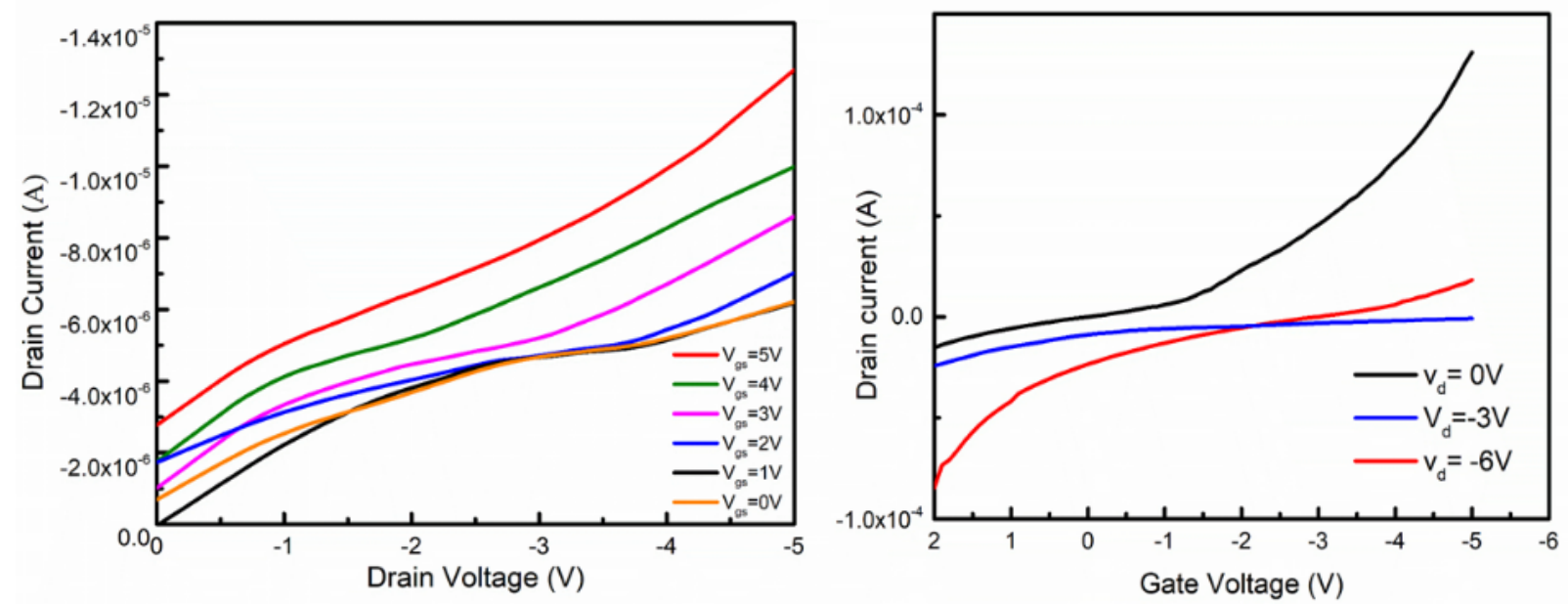

FIGURE 3. (a) shows the output characteristic and (b) shows the transfer characteristic of the $2 \mathrm{D}-\mathrm{MoS}_{2}$ based FET.

Optical properties of the prepared sample have been studied by UV-Visible absorption spectroscopy. The absorbance spectra is shown in the figure 2 (a). The UV-Visible absorption spectroscopy reveals 03 peak positions marked as A, B and C $(660 \mathrm{~nm}, 617 \mathrm{~nm}$ and $470 \mathrm{~nm}$ respectively) in spectra. The peaks A and B are due to the direct excitonic transition at the same k-point while peak $\mathrm{C}$ is due to the existence of von-hove singularity. The bandgap of the sample is calculated using Tau equation [10] and found to be $1.8 \mathrm{eV}$. Although, the I-V characteristic has been measured in very rough way (using the crocodile clips and patterned ITO), it gives the electrical signature of fabricated FET \{fig. 3 (a) and (b) \}. The output and transfer characteristics of device indicate that the drain current decreases as the negative voltage is increased due to the increase in the width of the depletion width. The observed current on/off ratio of the device is $10^{4}$.

\section{CONCLUSIONS}

In summary, $2 \mathrm{D}-\mathrm{MoS}_{2}$ nanosheets have been synthesized successfully by liquid phase exfoliation method having lateral dimensions upto few $\mu \mathrm{m}$ and 4 layers thick and bandgap of $1.8 \mathrm{eV}$. The top-gate type FET device have been fabricated and found that current on/off ratio is $10^{4}$. 


\section{ACKNOWLEDGMENTS}

Authors (RS, MC and OPS) would like to acknowledge the DRDO-SSPL for financial support. Also authors are thankful to IUAC for extending FESEM facilities, funded by the MoES under Geochronology project.

\section{REFERENCES}

1. H. Qiu, L. Pan, Z. Yao, J. Li, Y. Shi, and X. Wang, "Electrical characterization of back-gated bi-layer MoS 2 field-effect transistors and the effect of ambient on their performances," Appl. Phys. Lett., 2012.

2. X. Li, X. Wang, L. Zhang, S. Lee, and H. Dai, "Chemically derived, ultrasmooth graphene nanoribbon semiconductors," Science (80-. )., 2008.

3. M. Y. Han, B. Özyilmaz, Y. Zhang, and P. Kim, "Energy band-gap engineering of graphene nanoribbons," Phys. Rev. Lett., 2007.

4. H. S. S. Ramakrishna Matte et al., "MoS2 and WS2 analogues of graphene," Angew. Chemie - Int. Ed., 2010.

5. S. Ahmed and J. Yi, "Two-Dimensional Transition Metal Dichalcogenides and Their Charge Carrier Mobilities in Field-Effect Transistors," Nano-Micro Lett., 2017.

6. X. Tong, E. Ashalley, F. Lin, H. Li, and Z. M. Wang, "Advances in MoS 2 -Based Field Effect Transistors (FETs)," Nano-Micro Letters. 2015.

7. B. Radisavljevic, A. Radenovic, J. Brivio, V. Giacometti, and A. Kis, "Single-layer MoS2 transistors," Nat. Nanotechnol., 2011.

8. R. J. Smith et al., "Large-scale exfoliation of inorganic layered compounds in aqueous surfactant solutions," Adv. Mater., 2011.

9. H. Li et al., "From bulk to monolayer MoS 2: Evolution of Raman scattering," Adv. Funct. Mater., 2012.

10. V. Forsberg et al., "Exfoliated MoS2 in Water without Additives," PLoS One, 2016. 